\title{
Letter
}

\section{A Personal Perspective on Some Issues Relating to New Recommendations of the ICRP}

\author{
Andrew C. McEWAN*1
}

(Received on September 12, 2002)

\section{INTRODUCTION}

Historically the ICRP has revised its Recommendations for radiation protection every 10-15 years. Attention has commonly been focussed on dose limits and over the more than 70 years the ICRP has been in existence the dose limits for occupational exposure have been revised several times, to take account of the changing types of radiation in use and to reflect both changed views of the tolerability of risk and revised estimates of the risk of stochastic effects. However, the system of protection in scope and application is much wider than limits; indeed in the 1990 Recommendations ${ }^{1)}$ limits are seen as bounds to exposure with most emphasis being placed on keeping exposures "as low as reasonably achievable, social and economic factors being taken into account" (ALARA), or optimisation.

A Task Group of the Main Commission is currently developing a framework for revised Recommendations ${ }^{2)}$. In reviewing the Recommendations the Commission has taken note of areas where the current Recommendations have been poorly understood or applied. One of these is in the distinction between practices and interventions. Regulatory agencies in some countries do not appear to recognise the distinction and there are a number of examples of dose limits applicable to practices having being used in intervention situations. Another area for disquiet about the application of the 1990 Recommendations has been the way in which collective dose has been used both in the estimation of health effects in populations and in the process of optimisation. The Task Group has widely signalled its intention to repackage the presentation of the system of protection to make it more readily understood and applied. In doing so it has also indicated that greater emphasis will be placed on protection of individuals so that the limitation principle will be first applied in protection

\footnotetext{
${ }^{* 1}$ Former Director, National Radiation Laboratory; 24 Ranfurly St, Christchurch, New Zealand.
}

evaluations, then optimisation of individual protection.

\section{NEW TERMS}

The re-presentation of the system of protection is being accomplished by the introduction of several new terms and the setting aside of a number of current ones. Changing terminology always has some cost attached to it and clear benefits need to be seen before changes are cemented in. In avoiding the terms practices and interventions the concepts of controllable sources and uncontrollable sources have been mooted. However, the situations where these are employed generally correspond to those for practices and interventions, respectively. The distinction between the two is being de-emphasised, however, in that Protective Action Levels (PALs), or Doses for Protective Action, are being applied to both. Protection Action Levels are a new term for dose limits, constraints, intervention action and other levels. It is suggested in the outline of the Task Group thinking ${ }^{2}$ that the PALs (with different quantities and numeric values for different situations) would largely replace the range of limit and level terms that are in current use and Recommendations. However, the PALs require to be identified with the context in which they are to be applied, e.g. the PAL for annual dose to designated workers, or the PAL for discharge from a facility. While a semblance of unification may be achieved in the use of a common term covering all situations, the number of qualifying phrases to identify each context or situation could be expected to approximate the number of dose limit and level terms in current use.

One potential complexity or confusion in the use of the term "uncontrollable source" is that sources that are "unamenable to control" are discussed in the 1990 Recommendations in a somewhat different context. Such sources, which include cosmic radiation, natural distributions of primordial radionuclides and potassium- 40 and other natural emitters in the body, are considered to be outside the scope of the Recommendations since no 
practical measures can alter exposures arising from them. The non-controllable sources now being discussed appear to include these but also those for which interventions can modify exposures.

Other terms introduced in the Special Issue report ${ }^{2)}$ are "minimum level of protection" and "optimum level of protection". The minimum level of protection can be seen to be the dose limit, constraint, or PAL values, while the optimum level of protection corresponds to the doses resulting after a process of optimisation, i.e. the reduction of doses below the PAL values to the lowest reasonably achievable. A question to be answered is whether these terms, if retained, aid clarification of the currently understood concepts of limitation and optimisation in practices, and optimisation of averted dose below intervention action levels for interventions.

\section{NATURAL SOURCES}

The outline paper does not specifically consider natural sources of radiation other than to suggest an intervention level (PAL) for radon in dwellings. In previous Recommendations natural emitters have been treated rather differently from man-introduced nuclides, and this separation appears set to continue, reflecting both the different perspective in which natural radiation is viewed in the wider community and the general difficulty of subjecting it to control. One fundamental reason for different limits being applicable is that, apart from mining and milling of uranium and thorium ores, generally natural radiation sources arise 'adventitiously'. They therefore give rise to a chronic exposure situation for which intervention is required if doses are to be reduced, and as a consequence limits and control measures for practices are not applicable. In discussion of controls for natural sources that the situation is one of intervention seems often to be overlooked.

Greater emphasis is being given in the revised Recommendations to presenting the PALs in relation to natural background levels. Common variations in annual background dose lie within the range $1-10 \mathrm{mSv} / \mathrm{y}$, and the estimated mean value to the world population of $2.4 \mathrm{mSv} / \mathrm{y}$ is being used as a yardstick with which to compare the PAL values.

In ICRP Publication $65^{3)}$ the Action Level for radon in dwellings was to be set at a radon concentration in the range 200 to $600 \mathrm{~Bq} \mathrm{~m}^{-3}$, equivalent to an average annual effective dose, as derived from epidemiological studies, of 3 to $10 \mathrm{mSv}$. The corresponding action level for workplaces was in the range 500 to $1,500 \mathrm{~Bq} \mathrm{~m}^{-3}$, also equivalent to an average annual effective dose of 3 to $10 \mathrm{mSv}$. If, in new Recommendations, a single action level (PAL) value were proposed, it is not entirely clear whether the intention is that intervention would be taken where the PAL is exceeded, and no action taken if it is not, or whether action would be always indicated for levels exceeding the PAL and optimisation carried out to achieve exposures as low as reasonably achievable. In giving a range of values in ICRP Publication 65, authorities had a ready basis for introducing different requirements for new and existing buildings, or for deciding on a level where intervention should always be undertaken, and a level below which intervention need not be undertaken. Further thought on how the PAL value or values for radon are to be applied seems to be needed.

There appears to be a potential for different treatment of radon and other sources of natural exposure. Currently there is a degree of confusion as to if and when regulatory controls are to be imposed on natural radiation sources, other than radon, although international guidance is available in ICRP Publication $75^{4)}$ and the IAEA Safety Guide RS-G-1.15). The latter, in particular, proposes that the approach to controls on radon could be extended to elevated gamma radiation dose rates. Annual effective dose rates of say, $3 \mathrm{mSv}$, corresponding to the low end of the range for radon exposures, might be used as an action level or PAL, with doses below this level exempt from regulatory controls, and doses above the PAL to be treated as a practice, unless exempted on grounds such as unamenability to control. (The proposed value of $3 \mathrm{mSv}$ makes some allowance for the uncertainty in the dosimetry for radon and also that in the case of radon the inhalation dose is delivered almost entirely to a single organ).

Aircrew are exposed to cosmic radiation doses in the range $2-6 \mathrm{mSv} / \mathrm{y}$, and generally towards the lower end of the range. It is acknowledged the doses received by aircrew are not amenable to control by any reasonable means. The Commission might therefore consider returning to the position of ICRP Publication 26 where cosmic radiation exposure of aircrew in sub-sonic aircraft was viewed as not amenable to control and therefore outside the scope of the Commission's Recommendations ${ }^{6}$.

\section{OTHER ISSUES}

It is widely recognised that stability of the ICRP Recommendations is desirable in view of the long time periods required to adjust international standards and guidance, and to amend national legislations. Areas of potential change are in the definition of dose quantities. While some changes in dose quantities would appear desirable for the radiation weighting factors applied to neutrons and protons, other changes such as in the number of organs for which tissue weighting factors are specified, will have no material effect on the standards of radiation protection and would require extensive re-calculation of all dose quantities. Another potential change is in the occupational dose limit. It could be argued that standards of safety in other occupations have improved over time and the risk to someone occupationally exposed at the current dose limit over a working life poses a risk which is now large in comparison with those for other occupational hazards. However, the risk expression from 
uniform exposure to radiation throughout a working life is sharply peaked at late ages and not comparable in terms of loss of life and socio-economic impact with prompt fatalities occurring from industrial accidents. Any lowering of the current occupational limit would convey a message that radiation is again more hazardous than previously thought, when in fact there would appear to be no new epidemiological evidence to support this. Such a change at this time would appear detrimental to radiation use applications.

\section{CONCLUSIONS}

The re-presentation of the Recommendations currently being addressed may aid in achieving a more consistent application of the Commission's Recommendations. The new terms and universal application of PALs will be worth it if this can be achieved. It is highly desirable that stability of the Recommendations be maintained; any changes that are not of importance for radiation protection should be resisted. In particular a change in the occupational dose limit would appear to have a detrimental outcome for applications involving the use of radiation.

\section{REFERENCES}

1) International Commission on Radiological Protection; 1990 Recommendations of the International
Commission on Radiological Protection, Publication No. 60, Pergamon Press, Oxford and New York (1991).

2) R.H. CliRKE; Radiological protection at the start of the 21st century: a progress report, Jpn. J. Health Phys., 37 (1), 56-58 (2002).

3) International Commission on Radiological Protection; Protection against radon-222 at home and at work, Publication No. 65, Pergamon Press, Oxford and New York (1993).

4) International Commission on Radiological Protection; General principles for the radiation protection of workers, Publication No. 75, Pergamon Press, Oxford and New York (1997).

5) International Atomic Energy Agency; Occupational radiation protection: Safety Guide No.RS-G-1.1, International Atomic Energy Agency, Vienna (1999).

6) A.C. McEwAN; Is cosmic radiation exposure of air crew amenable to control? Radiat. Prot. Australas., 16(3), 21-25 (1999).

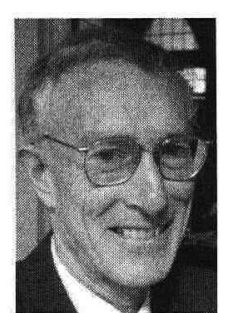

Andrew C. McEwan

$\mathrm{PhD}$, FRSNZ. Former Director of the New Zealand National Radiation Laboratory, Member of Committee 4 of the ICRP, and President of the Australasian Radiation Protection Society. 